\title{
A GEOMETRIC CRITERION FOR HYPERBOLICITY OF FLOWS
}

\author{
R. C. CHURCHILL, JOHN FRANKE AND JAMES SELGRADE
}

\begin{abstract}
A chain recurrent set for a flow on a compact manifold is hyperbolic if and only if it is quasi-hyperbolic. This result gives an easy proof that the geodesic flow on a compact manifold of negative curvature is hyperbolic.
\end{abstract}

1. Introduction. When studying structurally stable dynamical systems, a hypothesis of hyperbolicity, usually on the nonwandering set, is always assumed. Unfortunately, in practical problems (i.e., specific differential equations) this assumption is quite difficult to verify, even when the nonwandering set is only a periodic orbit [3]. In this paper, we present a simple geometric hypothesis for verifying hyperbolicity when the invariant set in question satisfies a weak recurrence condition known as chain recurrence. For diffeomorphisms a similar hypothesis has been studied by R. Mañé [10] and for vector bundle flows, by J. Selgrade [13] and also R. Sacker and G. Sell [12].

Consider a $C^{1}$-flow $f$ on a compact $C^{1}$-manifold $M$. Let $\Lambda \subset M$ be a closed invariant set for $f$ and let $T \Lambda$ denote $\left.T M\right|_{\Lambda} . \Lambda$ is said to be quasi-hyperbolic if

(i) the span $E$ of the vector field of $\left.f\right|_{\Lambda}$ is a subbundle of $T \Lambda$,

(ii) the flow on $T \Lambda / E$ induced by the tangent flow has no nontrivial bounded orbits.

Condition (i) guarantees that the fixed points in $\Lambda$ are isolated. Condition (ii) is easily seen to be equivalent to the following. Choose any Riemannian metric on $M$ and for $\varepsilon>0$ define $N(\varepsilon)=\left\{v \in T_{x} M\left|\inf _{\alpha \in R}\right| v-\alpha X(x) \mid<\varepsilon\right\}$, where $X$ is the vector field generating $f$. Then (ii) if and only if

(ii') there is an $\varepsilon>0$ such that $N(\varepsilon)$ contains no orbits other than those in $E$.

$\Lambda$ is said to be hyperbolic if the tangent flow $T f_{t}$ leaves invariant a continuous splitting $T \Lambda=E^{s} \oplus E^{u} \oplus E$ where, for some real numbers $c>0$ and $0<\lambda$ $<1$,

(i) if $v \in E^{u}$ and $t>0$ then $\left|T f_{-t}(v)\right| \leqslant c \lambda^{t}|v|$,

(ii) if $v \in E^{s}$ and $t>0$ then $\left|T f_{t}(v)\right| \leqslant c \lambda^{t}|v|$,

(iii) $E$ is the span of the vector field of $\left.f\right|_{\Lambda}$.

THEOREM. If $\left.f\right|_{\Lambda}$ is chain recurrent, then $\Lambda$ is hyperbolic if and only if $\Lambda$ is quasi-hyperbolic.

Received by the editors February 20, 1976.

AMS (MOS) subject classifications (1970). Primary 58F15, 34C35; Secondary 34C10, 34D30, $34 \mathrm{C} 40$.

(c) American Mathematical Society 1977 
In combination with results of J. Franke and J. Selgrade [7], [8], the result gives the existence of periodic orbits near $\Lambda$ and, in certain instances, transitive orbits within $\Lambda$. In fact, quasi-hyperbolicity of the maximal chain recurrent subset of $M$ is equivalent to Axiom $\mathrm{A}$ and the no cycle property.

As an application we will use this theorem to give an easy proof of a theorem of P. Eberlein [6], in the compact case, which includes the hyperbolicity of the geodesic flow on a compact Riemannian manifold of negative curvature.

2. Background and notation. Let $f$ be a flow on a compact metric space $M$. If $N$ is a subset of $M$ and $J$ a subset of $R$ then define $N \cdot J=f(N \times J)$. The $\omega$-limit set of $N$ is defined as

$$
\omega(N)=\bigcap_{t \geqslant 0} \mathrm{Cl}(N \cdot(t, \infty))
$$

A subset $A$ of $M$ is an attractor for $f$ if $A$ has a closed neighborhood $N$ such that $\omega(N)=A$. A repeller for $f$ is an atractor for the backwards flow. For each proper, nonempty attractor $A$ there is a maximal nonempty repeller in $M-A$ which is called the repeller dual to $A$. An attractor and its dual repeller will be referred to as an attractor-repeller pair.

Let $(E, M, \pi)$ denote a vector bundle over $M$ as defined in [2], where $E$ is the total space and $\pi$ is the projection onto $M$. By replacing each fiber of $E$ with the corresponding projective space, a fiber bundle $(P E, M, P \pi)$ is obtained (see $\S 2$ in [13]) and is called the projective bundle associated with $(E, M, \pi)$. The projective bundle retains the linearity of the vector bundle and also is compact. The pair $(F, f)$ will denote a vector bundle flow on $(E, M, \pi)$, i.e., $F_{t}$ is linear on the fibers of $E, f$ is a flow on $M$, and $\pi \circ F_{t}=f_{t} \circ \pi$ for all $t \in R$. Because of the linearity of $F_{t}$, it induces a flow on $P E$ denoted by $P F$. For our purposes $E$ will be the tangent bundle of a differentiable manifold or a quotient bundle of the tangent bundle, and $F$ will be the tangent flow or one induced by the tangent flow on an appropriate quotient bundle.

Given $x, y \in M$ and $\varepsilon, T>0$, an $(\varepsilon, T)$-chain from $x$ to $y$ is a set $\left\{x_{0}=x, x_{1}, \ldots, x_{k}=y ; t_{0}, \ldots, t_{k-1}\right\}$ so that for $0 \leqslant i \leqslant k-1$ we have $t_{i}$ $\geqslant T$ and $d\left(x_{i} \cdot t_{i}, x_{i+1}\right)<\varepsilon$. A point $x$ is chain equivalent to $y$ (written $x \sim y$ ) if for any $\varepsilon, T>0$ there is a $(\varepsilon, T)$-chain from $x$ to $y$ and one from $y$ to $x$. The chain recurrent set $\mathscr{R}(f)$ is $\{x \in M \mid x \sim x\}$. $R(f)$ is a closed invariant set containing the nonwandering set. For $\Lambda \subset M$ we say $\left.f\right|_{\Lambda}$ is chain recurrent if $\Lambda$ is a compact invariant set and $\Re\left(\left.f\right|_{\Lambda}\right)=\Lambda$. C. Conley [4] shows that $\left.f\right|_{\mathscr{R}(f)}$ is chain recurrent.

The next two theorems follow from results in [13]:

THEOREM 2.1. Suppose $(F, f)$ is a vector bundle flow on $(E, M, \pi)$ with associated flow $P F$ on $P E$. If $f$ is chain recurrent on $M$, then each attractorrepeller pair for PF corresponds to a continuous $F$-invariant splitting of $E$. 
THEOREM 2.2. Let $(F, f)$ be a vector bundle flow on $(E, M, \pi)$ and $f$ be chain recurrent on $M$. If no nontrivial orbits of $F$ are bounded, then $E$ is the direct sum of two invariant subbundles (one possibly empty), one contracting exponentially in positive time and the other in negative time (see (i) and (ii) in the definition of hyperbolic).

3. Main result. In this section $M$ is a compact $C^{1}$-manifold with a Riemannian metric and $f$ a $C^{1}$-flow on $M .(T M, M, \pi)$ is the tangent bundle with tangent flow $\left(T f_{t}, f_{t}\right)$, and $(P T M, M, P \pi)$ is the associated projective bundle with flow $P T f_{t}$. Let $P: T M \rightarrow P T M$ denote the natural identification map. The Riemannian metric on $M$ induces a topological metric on PTM which, on each fiber, measures the sine of the angle between two lines (see $\$ 2$ in [13]).

Let $\Lambda$ denote a closed invariant subset of $M$ and $E$ denote the span of the vector field of $\left.f\right|_{\Lambda}$. If $E$ is a subbundle of $T \Lambda=\left.T M\right|_{\Lambda}$, then $E^{\perp}$ denotes the orthogonal complement of $E$ in $T \Lambda$ with respect to the Riemannian metric on $M$. For $v \in T \Lambda, e(v)$ and $e^{\perp}(v)$ denote the projections of $v$ onto $E$ and $E^{\perp}$, respectively. Let $\sigma: T \Lambda \rightarrow T \Lambda / E$ denote the quotient map and $[v]$ $\in T \Lambda / E$ denote $\sigma(v)$ for each $v \in T \Lambda$. There is a norm $\|\cdot\|$ on $T \Lambda / E$ induced by the Riemannian metric on $M$, i.e., $\|[v]\| \equiv\left|e^{\perp}(v)\right|$ for $v \in T \Lambda$.

In this setting we state and prove our main result.

THEOREM 3.1. If $\left.f\right|_{\Lambda}$ is chain recurrent, then $\Lambda$ is hyperbolic if and only if $\Lambda$ is quasi-hyperbolic.

Proof. Clearly hyperbolicity implies quasi-hyperbolicity so we show the opposite implication.

Condition (i) in the definition of quasi-hyperbolic implies that the fixed points of $\Lambda$ are isolated and condition (ii) implies that the tangent flow above them has no nontrivial bounded orbits. Thus Theorem 2.2 gives that the fixed points are hyperbolic. So without loss of generality assume $\Lambda$ has no fixed points.

Since the induced flow on $T \Lambda / E$ has no nontrivial bounded orbits, apply Theorem 2.2 to get a continuous invariant splitting of $T \Lambda / E, T \Lambda / E=E^{+}$ $\oplus E^{-}$, where $E^{+}$is contracting exponentially in positive time and $E^{-}$in negative time. Consider $E^{+}$. There is a $\lambda \in(0,1)$ and $T>0$ such that for each $[\imath] \in E^{+}$,

$$
\left\|\left[T f_{t}(v)\right]\right\| \leqslant \lambda^{t}\|[v]\| \quad \text { for all } t \geqslant T \text {. }
$$

Let $F^{s} \subset T \Lambda$ denote $\sigma^{-1}\left(E^{+}\right)$. Then $F^{s}$ is an invariant subbundle containing $E$. The previous inequality asserts that for each $v \in F^{s}$,

$$
\left|e^{\perp}\left(T f_{t}(v)\right)\right| \leqslant \lambda^{t}\left|e^{\perp}(v)\right| \text { for all } t \geqslant T .
$$

We need to find an invariant complement to $E$ in $F^{s}$. (Notice $E^{\perp}$ is not necessarily invariant.) On such a complement another application of Theorem 
2.2 will give the exponential contraction of $T f_{t}$. To obtain this complement we pass to the projective bundle $P T M$ and show that $P E$ is an attractor in $P F^{s}$. The inverse image under $P$ of its dual repeller will be the desired complement. To show $P E$ is an attractor, we will find a neighborhood $N$ of $P E$ in $P F^{s}$ so that an arbitrarily small neighborhood of $P E$ contains $N \cdot(t, \infty)$ for large $t$, and thus $\omega(N)=P E$. Since the distance in $P T M$ is deterrnined by the sine of the angle between lines, the neighborhood $N$ will be shown to consist of lines determined by vectors $v \in F^{s}$ such that the ratio $\left|e^{\perp}(v)\right| /|e(v)|$ is small and such that the ratio $\left|e^{\perp}\left(T f_{t}(v)\right)\right| /\left|e\left(T f_{t}(v)\right)\right|$ is small for large $t$.

Since the vector field of $f$ is bounded and nonzero on $\Lambda$, there are positive constants $\alpha \geqslant 1$ and $\beta$ so that for all $v \in E$ and $t \in R$,

$$
\beta|v| \leqslant\left|T f_{t}(v)\right| \leqslant \alpha|v| .
$$

By compactness and the linearity of $T f_{t}$, there are positive constants $A$ and $B$ such that for all $v \in F^{s}$ and $t \in[T, 2 T]$,

$$
B|v| \leqslant\left|T f_{t}(v)\right| \leqslant A|v| .
$$

Fix $t \in[T, 2 T]$. Because of exponential contraction in the direction normal to $E$, for each positive integer $n$,

$$
\left|e^{\perp}\left(T f_{n t}(v)\right)\right| \leqslant \lambda^{n t}\left|e^{\perp}(v)\right| .
$$

Bounding $\left|e\left(T f_{n t}(v)\right)\right|$ below is more complicated because the component of $v$ normal to $E$ may "turn" in the $E$ direction to give a component in the $E$ direction that may reduce $\left|e\left(T f_{t}(e(v))\right)\right|$. We show that under iteration the total contribution in the $E$ direction caused by the "turning" is finite and, thus, by choosing $|e(v)|$ large initially, $\left|e\left(T f_{n t}(v)\right)\right|$ can be bounded below.

Define $w_{k}$ recursively by

$$
w_{0}=v, \quad w_{k}=T f_{t}\left(e^{\perp}\left(w_{k-1}\right)\right) \quad \text { for } k=1, \ldots, n .
$$

Thus by using (2) and then (3)

$$
\left|w_{k}\right| \leqslant A\left|e^{\perp}\left(w_{k-1}\right)\right| \leqslant A \lambda^{t}\left|e^{\perp}\left(w_{k-2}\right)\right| \leqslant \cdots \leqslant A \lambda^{(k-1) t}\left|e^{\perp}(v)\right| .
$$

So for $k=1, \ldots, n$,

$$
\left|w_{k}\right| \leqslant A \lambda^{(k-1) t}\left|e^{\perp}(v)\right|
$$

Notice that for each poistive integer $n$,

$$
T f_{n t}\left(e^{\perp}(v)\right)=w_{n}+\sum_{k=1}^{n-1} T f_{k t}\left(e\left(w_{n-k}\right)\right) .
$$

Thus, using (1), (4) and (5), 


$$
\begin{aligned}
\left|e\left(T f_{n t}(v)\right)\right| & =\left|e\left(T f_{n t}(e(v))\right)+e\left(T f_{n t}\left(e^{\perp}(v)\right)\right)\right| \\
& \geqslant\left|e\left(T f_{n t}(e(v))\right)\right|-\left|e\left(T f_{n t}\left(e^{\perp}(v)\right)\right)\right| \\
& \geqslant \beta|e(v)|-\left|w_{n}\right|-\sum_{k=1}^{n-1}\left|T f_{k t}\left(e\left(w_{n-k}\right)\right)\right| \\
& \geqslant \beta|e(v)|-A \lambda^{(n-1) t}\left|e^{\perp}(v)\right|-\sum_{k=1}^{n-1} \alpha\left|w_{n-k}\right| \\
& \geqslant \beta|e(v)|-A \lambda^{(n-1) t}\left|e^{\perp}(v)\right|-\alpha \sum_{k=1}^{n-1} A \lambda^{(k-1) t}\left|e^{\perp}(v)\right| \\
& \geqslant \beta|e(v)|-\alpha A\left|e^{\perp}(v)\right| \sum_{k=1}^{n} \lambda^{(k-1) t} \\
& \geqslant \beta|e(v)|-\alpha A\left|e^{\perp}(v)\right|\left(1-\lambda^{t}\right)^{-1} .
\end{aligned}
$$

The neighborhood $N$ of $P E$ is chosen so that for all $[v] \in N$, the ratio $\left|e^{\perp}(v)\right| /|e(v)|$ is small enough to guarantee that when $|v|=1$,

$$
\beta|e(v)|-\alpha A\left|e^{\perp}(v)\right|\left(1-\lambda^{T}\right)^{-1}
$$

is larger than some fixed positive number. From (3) and (6), for each $t \in[T, 2 T]$

$$
\frac{\left|e^{\perp}\left(T f_{n t}(v)\right)\right|}{\left|e\left(T f_{n t}(v)\right)\right|} \leqslant \frac{\lambda^{n T}\left|e^{\perp}(v)\right|}{\beta|e(v)|-\alpha A\left|e^{\perp}(v)\right|\left(1-\lambda^{T}\right)^{-1}} .
$$

Given $\varepsilon \geqslant 0$ this ratio can be made smaller than $\varepsilon$ for all $[v] \in N$ by choosing $n$ large. Thus, for large $n, N \cdot(n T, \infty)$ is contained within an $\varepsilon$ neighborhood of $P E$ and so $\omega(N)$ is within an $\varepsilon$ neighborhood of $P E$. Since $\varepsilon$ is arbitary $\omega(N)=P E$ and so $P E$ is an attractor in $P F^{s}$. Let $G$ be the repeller dual to $P E$. Thus $P E$ and $G$ are an attractor-repeller pair for $P T f_{t}$ in $P F^{s}$. By Theorem 2.1, $E^{s} \equiv P^{-1}(G)$ is a $T f_{t}$ invariant subbundle of $F^{s}$ such that $E^{s} \oplus E=F^{s}$. Since the orbits of $E^{+} \subset T \Lambda / E$ are unbounded in negative time and $E^{s}$ is invariant and bounded away from $E$, all orbits of $E^{s}$ are unbounded in negative time. Thus Theorem 2.2 implies the $E^{s}$ contracts exponentially in positive time.

The preceding argument applies to $E^{-} \subset T \Lambda / E$ and the backwards flow gives the existence of $E^{u}$ contracting exponentially in negative time. Clearly, $T \Lambda=E^{s} \oplus E^{u} \oplus E$ and the proof is complete.

4. An example. As an application of the hyperbolicity theorem we will give a quick proof of the following theorem of P. Eberlein [6]. Let $M$ be a compact Riemannian manifold, $S M$ the unit sphere bundle, and $f$ the geodesic flow on $S M$.

THEOREM 4.1. SM is hyperbolic if and only if there exists no nonzero orthogonal 
Jacobi field $J$ along a unit speed geodesic of $M$ such that $|J(t)|$ is bounded for all $t$.

If $M$ has negative curvature, then verifying the unboundedness of orthogonal Jacobi fields is done exactly as is the proof of the nonexistence of conjugate points (e.g., see [11, p. 100]). The possibility of applying Theorem 2.2 to give a new proof of the hyperbolicity of these geodesic flows was first pointed out to us by $\mathrm{C}$. Chicone.

Eberlein does not assume the compactness of $M$, but does assume the nonexistence of conjugate points and completeness. However, as pointed out by Eberlein, Klingenberg has shown that if a geodesic flow is hyperbolic, then there can be no conjugate points [9].

To prove the theorem we need the following standard facts concerning Jacobi fields and orbits of the induced flow Tf on TSM. We let $\pi_{1}: S M$ $\rightarrow M$ and $\pi_{2}: T S M \rightarrow S M$ be the usual bundle projections.

(i) Given a geodesic $x=x(t)$ on $M$, every Jacobi field $J$ along $x$ can be written uniquely as

$$
J=J^{\perp}+a \dot{x}+b t \dot{x}
$$

where $J^{\perp}$ is an orthogonal Jacobi field and $a, b$ are constants.

(ii) There is a one-one correspondence between orbits $z(t)$ of $T f$ and those Jacobi fields along the geodesic $x(t)=\pi_{1}\left(\pi_{2}(z(t))\right)$ with $|\dot{x}| \equiv 1$ and $b=0$ in (1).

(iii) The Riemannian metric on $M$ can be lifted to one on $S M$ in such a way that:

(a) $E^{\perp}$ is $T$-invariant; and

(b) if $z(t) \in E^{\perp}$, then the corresponding (via (ii)) Jacobi field $J_{z}$ is orthogonal, and

$$
|z(t)|=\left|J_{z}(t)\right|, \quad t \in R .
$$

Proofs of (i)-(iii) can be found, for example, in [1, §22], or [6, pp. 440-441]. The geodesic flow on $T M$ is conjugate, via the Legendre transformation, to a Hamiltonian flow on $T^{*} M$. It follows by Liouville's theorem that there is a volume preserved on energy surfaces, hence the flow is nonwandering, and thus chain recurrent, on $S M$. Eberlein's result, as we have stated it, is then immediate from (2), the unboundedness assumption on orthogonal Jacobi fields, and Theorem 3.1.

In the case of a geodesic flow the bundles $E^{s}$ and $E^{u}$ both have the same dimension, necessarily $n-1$ if $\operatorname{dim} M=n$. In fact equality of dimensions will always be the case when the flow can be regarded as Hamiltonian. To see this let $\tilde{E}^{s}, \tilde{E}^{u}, \tilde{E}$ denote the bundles over $S^{*} M$ corresponding to $E^{s}, E^{u}, E$ via the Legendre transformation, and let $\omega$ denote the canonical two-form on $T^{*} M$. Then $\omega$ is preserved by the flow and bounded on the unit disk bundle. The growth and decay in $E^{u}, E^{s}$ and the boundedness of the vector field of $f$ imply that $\omega$ vanishes on $\tilde{E}^{s} \oplus \tilde{E}, \tilde{E}^{u} \oplus \tilde{E}$. But then $\tilde{E}^{s} \oplus \tilde{E}, \tilde{E}^{u} \oplus \tilde{E}$ intersect 
fibres in isotropic subspaces, which can have dimension at most $n$ (see any reference on Lagrangian subspaces, e.g., Chapter 2 of [5]). Since $\operatorname{dim} \tilde{E}^{s} \oplus \tilde{E}^{u}$ $=2 n-2$ and $\operatorname{dim} \tilde{E}=1$, this forces $\operatorname{dim} \tilde{E}^{s}=\operatorname{dim} \tilde{E}^{u}=\operatorname{dim} E^{s}=\operatorname{dim} E^{u}$ $=n-1$.

J. Franke and J. Selgrade have shown that hyperbolicity and chain recurrence on the entire manifold imply the existence of transitive orbits and the denseness of periodic orbits [7], [8]. Thus we obtain new proofs of these properties of geodesic flows which satisfy Eberlein's hypotheses.

\section{REFERENCES}

1. D. V. Anosov, Geodesic flows on closed Riemann manifolds with negative curvature, Trudy Mat. Inst. Steklov 90 (1967); English transl., Amer. Math. Soc., Providence, R. I., 1969. MR 36 \# 7157; 39 \#3527.

2. M. Atiyah, $K$-theory, 2nd ed., Benjamin, New York, 1967. MR 36 \#1130.

3. R. C. Churchill, G. Pecelli and D. L. Rod, Hyperbolic periodic orbits, J. Differential Equations (to appear).

4. C. C. Conley, The gradient structure of a flow: I, IBM Research, RC 3932 (\#17806), Yorktown Heights, New York, July 17, 1972.

5. J. J. Duistermaat, Fourier integral operators, Courant Inst. of Mathematical Sciences, New York Univ., New York, 1973.

6. P. Eberlein, When is a geodesic flow of Anosov type?. I, J. Differential Geometry 8 (1973), 437-463.

7. J. Franke and J. Selgrade, Hyperbolicity and chain recurrence, J. Differential Equations (to appear).

8. , Abstract $\omega$-limit sets, chain recurrent sets, and basic sets for flows, Proc. Amer. Math. Soc. (to appear).

9. W. Klingenberg, Riemannian manifolds with geodesic flow of Anosov type, Ann. of Math. (2) 99 (1974), 1-13.

10. R. Mañé, Persistent manifolds are normally hyperbolic, Bull. Amer. Math. Soc. 80 (1974), 90-91. MR 49 \# 4043.

11. J. W. Milnor, Morse theory, Princeton Univ. Press, Princeton, N. J., 1963. MR 29 \#634.

12. R. J. Sacker and G. R. Sell, Existence of dichotomies and invariant splittings for linear differential systems. I, J. Differential Equations 15 (1974), 429-458. MR 49 \#6209.

13. J. F. Selgrade, Isolated invariant sets for flows on vector bundles, Trans. Amer. Math. Soc. 203 (1975), 359-390.

Department of Mathematics, Hunter College, CUNY, New York, New York 10021 (Current address of R. C. Churchill)

Department of Mathematics, North Carolina State University, Raleigh, North CaroLINA 27607 (Current address of John Franke and James Selgrade) 